\title{
Minimally Invasive Surgery for Congenital Abdominal Cystic Lesions in Newborns and Infants
}

\author{
PRZEMYSŁAW GAŁĄZKA ${ }^{1,2}$, KRZYSZTOF REDLOCH $^{3}$, KACPER KROCZEK $^{1,2}$ and JAN STYCZYŃSKI ${ }^{2}$ \\ ${ }^{1}$ Department of General and Oncological Surgery for Children and Adolescents \\ with Unit of Neonatal Surgery and Intensive Care, Nicolaus Copernicus University in Torun, \\ Ludwik Rydygier Collegium Medicum, Bydgoszcz, Poland; \\ ${ }^{2}$ Department of Pediatric Hematology and Oncology, Nicolaus Copernicus University in Torun, \\ Ludwik Rydygier Collegium Medicum, Bydgoszcz, Poland; \\ ${ }^{3}$ Department of Radiology, Nicolaus Copernicus University in Torun, \\ Ludwik Rydygier Collegium Medicum, Bydgoszcz, Poland
}

\begin{abstract}
Background/Aim: In the variety of congenital abdominal cystic lesions (CACL) of different origin, ovarian cyst is the most common intra-abdominal pathology in female neonates. The prognosis and timing of treatment varies depending on the nature of CACL. This study aimed to assess the results of diagnostics and treatment of CACL. Patients and Methods: A retrospective analysis was performed of 39 cases of CACL, with the spectrum including ovarian, enteric, mesenteric and pancreatic origin. Outcome of minimally invasive surgery, open surgery or conservative approach was analyzed. Results: Twenty-eight neonates underwent surgery, while 11 were treated conservatively. Twenty patients were treated with a laparoscopic technique and eight with laparotomy combined with laparoscopy. Final diagnosis included: Fifteen cases of ovarian pathology (ovarian torsion in 11 cases), 12 treated laparoscopically and three with laparotomy, six enteric duplications (four laparoscopic and two laparotomic), three mesenteric cysts (one laparoscopic and two laparotomic), two pancreatic cysts (both laparoscopic only), two duodenal stenoses, including duodenal septum (both laparotomies with HeinekeMikulicz plasty). No blood transfusion apart from two cases requiring re-laparotomy and no early complications were
\end{abstract}

This article is freely accessible online.

Correspondence to: Przemysław Gałązka MD, Ph.D., Department of General and Oncological Surgery for Children and Adolescents, Jurasz University Hospital, Nicolaus Copernicus University Toruń, Collegium Medicum, ul. Sklodowskiej-Curie 9, 85-094 Bydgoszcz, Poland. Tel: +48 525854015, Fax: +48 525854093, e-mail: galazkaprzemek@hotmail.com

Key Words: Congenital abdominal cystic lesions, newborns, infants, algorithm. observed in any case; no death occurred. Conclusion: With the strategy of management based on ultrasound and laboratory data, a laparoscopically assisted minimal access approach resulted in minimal risk of complications and complete recovery in all patients, leading to exclusion of oncological risk.

Congenital abdominal cystic lesions (CACL) can be defined as non-solid lesions located in the abdominal cavity. CACL in neonates are not common, affecting about one in 1,500 live births (1). This diagnosis rate is however increasing due to better quality and experience during standard prenatal ultrasound and routine sonographic screening of newborns. Although ultrasound identification of a CACL is easy, determining its origin and nature can be challenging (2). There is a variety of CACL origins (Table I), with ovarian cyst being the most common intra-abdominal cyst in female neonates (1). Diagnostic and therapeutic management depends on the urgency of surgical treatment and possibilities of spontaneous resolution of the cyst, or on whether the timing of surgical treatment can be postponed under close follow-up. Such an approach determines the risk of post-surgery complications: the younger the child, the higher is the risk of potential morbidity and severe complications, while in later months of life, there is a better tolerance of applied surgical procedures, which are sometimes highly complicated for neonate $(3,4)$.

In diagnostics, ultrasound examination is typically the first-line modality, given its mobility, lack of ionizing radiation, low cost and high spatial and contrast resolution in small patients. Ultrasound in the neonatal period can often indicate the need for urgent surgical treatment or the possibility of a conservative strategy. Otherwise, computed tomographic imaging may be necessary in order to be able to choose the best management (1). 
Table I. Origin of congenital abdominal cystic lesions.

\begin{tabular}{|c|c|}
\hline System/organ & Cystic lesion \\
\hline Hepatobiliary & $\begin{array}{l}\text { Hepatic simple cyst } \\
\text { Choledochal malformation } \\
\text { Mesenchymal hamartoma } \\
\text { Ciliated foregut cyst } \\
\text { Epidermoid cyst }\end{array}$ \\
\hline Spleen & Splenic cyst \\
\hline Pancreas & Pancreatic cyst \\
\hline Urogenital & $\begin{array}{l}\text { Multi-cystic dysplastic kidneys } \\
\text { Hydrocolpos or hydrometrocolpos } \\
\text { Urachal cyst } \\
\text { Ovarian cyst (simple or complex) }\end{array}$ \\
\hline $\begin{array}{l}\text { Suprarenal } \\
\text { cystic lesions }\end{array}$ & $\begin{array}{l}\text { Neuroblastoma } \\
\text { Primary adrenal hemorrhage } \\
\text { Other rare adrenal cystic lesions (e.g. adrenal } \\
\text { cortical macrocysts, adrenal epithelial cysts, } \\
\text { pulmonary sequestrations) }\end{array}$ \\
\hline Gastrointestinal & $\begin{array}{l}\text { Enteric duplication cyst } \\
\text { Omphalo-mesenteric duct cyst (e.g. Meckel } \\
\text { diverticula) } \\
\text { Meconium pseudocyst }\end{array}$ \\
\hline Other & Cystic sacrococcygeal tumor \\
\hline
\end{tabular}

Based on Silva et al. (1).

The objective of this study was the analysis of surgical treatment of newborns and infants with CACL with the use of minimally invasive surgery based on a laparoscopically assisted minimal access (LAMA) approach and videoscopic assessment. Our study aimed to assess the results of diagnostics and treatment of CACL.

\section{Patients and Methods}

Design of the study. We retrospectively reviewed all cases of newborns and infants with CACL treated in our Neonatal and Pediatric Surgery Unit between 2011 and 2019. Out of a total number of 53 newborns and infants with CACL, 14 cases were excluded. Demographic data of the 39 patients included in the study are shown in Table II. This cohort was analyzed with respect to treatment approach; we analyzed the presence of risk factors and associated congenital anomalies, diagnostic data of ultrasound and other imaging modalities, laboratory data (alpha-fetoprotein, AFP; beta human chorionic gonadotropin, b-HCG; lactic dehydrogenase, $\mathrm{LDH})$, treatment focused on surgical approach, as well as the final outcome.

Exclusion criteria. Cases with an obvious extra-abdominal origin of the lesion and cases with urological anomalies such as multi cystic kidney disease or doubled collecting system, due to different diagnostic and therapeutic protocols, were excluded from the analysis.

Preoperative workup. Ultrasound examinations were performed by radiologists experienced in pediatric diagnosis. In the case of diagnostic doubts, computed tomography was performed. All cases
Table II. Demographic and clinical data of patients included in the study.

\begin{tabular}{|c|c|}
\hline Characteristic & Value \\
\hline \multicolumn{2}{|l|}{ Gender, n (\%) } \\
\hline Female & $29(74.4 \%)$ \\
\hline Male & $10(25.6 \%)$ \\
\hline \multicolumn{2}{|l|}{ Birth, n (\%) } \\
\hline Full-term & $30(76.9 \%)$ \\
\hline Premature & $9(23.1 \%)$ \\
\hline \multicolumn{2}{|l|}{ Gestational age, weeks } \\
\hline Mean (range) & $39(36-42)$ \\
\hline \multicolumn{2}{|l|}{ Birth weight, $\mathrm{kg}$} \\
\hline Mean (range) & $3.45(2.68-4.22)$ \\
\hline \multicolumn{2}{|l|}{ Caesarian section delivery, $\mathrm{n}(\%)$} \\
\hline Yes & $10 / 39(25.6 \%)$ \\
\hline \multicolumn{2}{|l|}{ Prenatal diagnosis of CACL, n (\%) } \\
\hline Yes & $30 / 39(76.9 \%)$ \\
\hline \multicolumn{2}{|l|}{ Maternal risk factors, $\mathrm{n}$} \\
\hline Hypothyroidism & 3 \\
\hline Diabetes mellitus & 2 \\
\hline Cholestasis & 1 \\
\hline Anemia & 1 \\
\hline \multicolumn{2}{|l|}{ Newborn co-morbidities, $\mathrm{n}$} \\
\hline Persistent foramen ovale & 4 \\
\hline ASD II & 2 \\
\hline Hyperbilirubinemia & 2 \\
\hline Hypotrophy & 2 \\
\hline Esophageal atresia & 1 \\
\hline Cleft palate & 1 \\
\hline Renal agenesis & 1 \\
\hline Capillary facial hemangioma & 1 \\
\hline CMV infection & 1 \\
\hline
\end{tabular}

CACL: Congenital abdominal cystic lesions; ASD: atria septum defect; CMV: cytomegalovirus.

were followed by the same unit and counseled by a team of pediatric surgeons, neonatologists and geneticists.

Ultrasound examination. All patients underwent postnatal abdominal ultrasound. In differential diagnostics of cystic structures, the following features were analyzed: Size, density, margins and walls, structure (mono- or polycystic), echogenicity and presence or absence of blood flow within the cystic wall. Usually, the walls of ovarian and mesenteric cysts are thin as opposed to duplication cysts which have stratified construction of the wall.

Treatment. Children were treated either surgically or conservatively. Surgical treatment included laparoscopy (endoscopic approach, minimally invasive surgery) or laparotomy (classic open surgery). The selection of approach was related to the following factors: Size of the cyst, clinical condition of the patient, weight of the patient, and comorbidities. The decision was team-dependent and undertaken according to an internal strategy (Figure 1).

Statistical analysis. Categorical variables are reported as numbers and percentages. Continuous variables were reported as medians with their range. Cumulative incidence of applied surgical treatment 


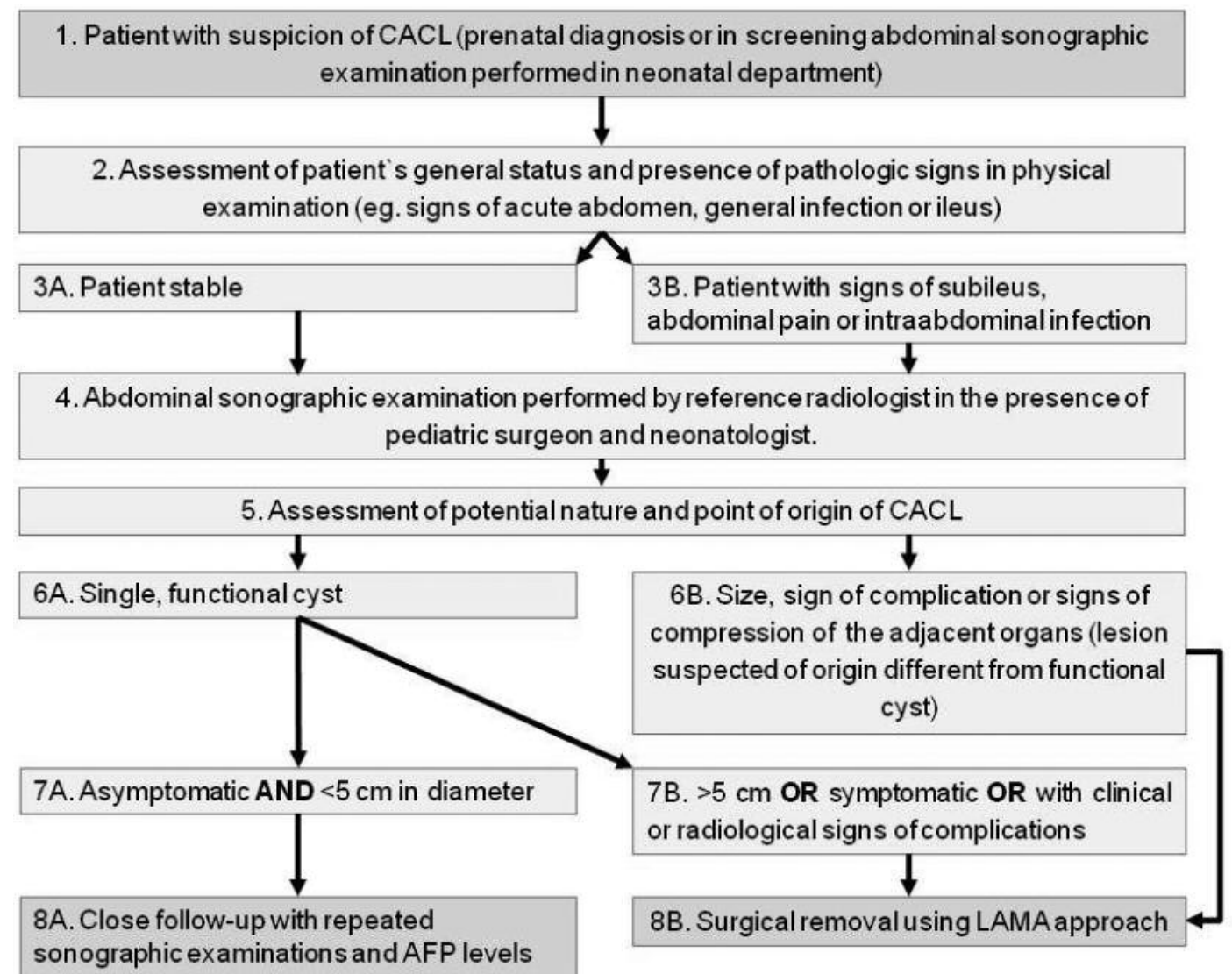

Figure 1. The strategy of diagnostic and therapeutic management of congenital abdominal cystic lesions (CACL). AF: Alpha fetoprotein; LAMA: laparoscopically assisted minimal access.

with respect to age of patient or time from hospitalization to surgery was calculated with the Kaplan-Meier method, and compared with log-rank test. A $p$-value of less than 0.05 was considered significant. SPSS 25 (IBM, Armonk, NY, USA) statistical package was used.

Ethical considerations. All investigations and treatments were established clinical practices and were carried out according to accepted clinical practice and in compliance with medical principles of the Declaration of Helsinki. Informed consent was obtained from all parents prior to treatment. In this retrospective case series, formal ethical approval was not required.

\section{Results}

Radiological and laboratory findings. In 29/39 (74.4\%) cases, prenatal diagnostics already indicated the presence of abdominal cystic structure. All children underwent ultrasound performed postnatally. In 9/39 (23.1\%) patients, computed tomographic imaging (or magnetic resonance imaging in one case) and laboratory data were also necessary for diagnosis. In all these patients, no abnormal results of oncological markers (AFP, bHCG, or LDH) were detected.
Clinical diagnosis and surgical management. In total, 28 neonates were assigned to surgical treatment, while the remaining 11 were treated conservatively. The majority $(n=20)$ were treated with a laparoscopic technique, and eight patients with laparotomy combined with laparoscopy (Table III).

Final clinical diagnosis which required surgical treatment included: 15 cases of ovarian pathology including ovarian torsion in 11 cases (12 treated laparoscopically and three with laparotomy), six enteric duplications (four laparoscopic and two laparotomic), three mesenteric cysts (one laparoscopic and two laparotomic), two pancreatic cysts (both laparoscopic only), two duodenal stenoses, including duodenal septum (both laparotomies with Heineke-Mikulicz plasty). Laparotomy was usually applied at a younger age than laparoscopy $(p=0.056)$ but the chosen method was not time-dependent with respect to the period from the day of hospitalization in the Surgical Department (Figure 2).

Surgical treatment. LAMA: All patients were operated on under general anesthesia. Urinary catheter was placed only while in surgery. Using the Hasson method, we created an 
Table III. Therapeutic management: Patient characteristics and outcome of therapy.

\begin{tabular}{|c|c|c|c|c|}
\hline Characteristic & $(n=39)$ & $\begin{array}{c}\text { Laparoscopy } \\
(\text { LAMA) }(n=20)\end{array}$ & $\begin{array}{l}\text { Laparotomy } \\
\qquad(\mathrm{n}=8)\end{array}$ & $\begin{array}{l}\text { Non-surgical } \\
\quad(\mathrm{n}=11)\end{array}$ \\
\hline \multirow[t]{11}{*}{ Diagnosis, $\mathrm{n}$} & Ovarian cyst $(\mathrm{n}=14)$ & 4 & 0 & 10 \\
\hline & Prenatal ovarian torsion $(\mathrm{n}=11)$ & 8 & 3 & 0 \\
\hline & Enteric duplication cyst $(\mathrm{n}=6)$ & 4 & 2 & 0 \\
\hline & Lymphatic mesenteric cyst $(n=3)$ & 1 & 2 & 0 \\
\hline & Pancreatic cyst $(n=2)$ & 2 & 0 & 0 \\
\hline & Congenital duodenal stenosis/septum $(n=2)$ & 1 & 1 & 0 \\
\hline & Splenic cyst $(n=1)$ & 0 & 0 & 1 \\
\hline & Prenatal diagnosis, n $(\%)$ & $14(70 \%)$ & $6(75 \%)$ & 10 \\
\hline & Postnatal ultrasound, n (\%) & $20(100 \%)$ & $8(100 \%)$ & $11(100 \%)$ \\
\hline & Computed tomography, n (\%) & $7(35 \%)$ & $1(12.5 \%)$ & 0 \\
\hline & Magnetic resonance imaging, $\mathrm{n}(\%)$ & $1(5 \%)$ & 0 & 0 \\
\hline Age at hospitalization, days & Median (range) & $6(1-210)$ & $2(1-13)$ & $3.5(2-12)$ \\
\hline Age at surgery, days & Median (range) & $8(2-212)$ & $4.5(2-15)$ & \\
\hline Hospitalization to surgery, days & Median (range) & $2(1-6)$ & $2.5(2-6)$ & \\
\hline Number of days to discharge & Median (range) & $5(2-17)$ & $8(3-13)$ & \\
\hline \multirow[t]{2}{*}{ Histopathology results } & Complex cyst (cystic tumor) & 11 & 3 & \\
\hline & Simple cyst & 9 & 5 & \\
\hline Other histopathology data & Enteric epithelium of cyst & 5 & 3 & \\
\hline \multirow[t]{3}{*}{ Surgical management } & Ovarectomy & 10 & 6 & \\
\hline & Cystectomy with enteric anastomosis & 5 & 2 & \\
\hline & Fenestration & 5 & 0 & \\
\hline Complications, n (\%) & Yes & $2(10 \%)^{*}$ & 0 & \\
\hline Reoperation, $\mathrm{n}$ & Yes & 2 & 0 & \\
\hline
\end{tabular}

LAMA: Laparoscopically assisted minimal access. *Both hemorrhage.

umbilical access for the first port and after insufflation to a pressure of $8 \mathrm{mmHg}$, a $5 \mathrm{~mm} 30^{\circ}$ scope was used. After initial assessment according to the nature of the lesion, further steps of the surgical procedure were conducted.

Ovarian cysts: These CACLs were the most frequent pathology, found in 25 females. In 15 cases, the diameter of cyst was $>5 \mathrm{~cm}$, being an indication for surgical treatment, which was laparoscopic in 12 cases and open classic laparotomy in three. In 11 cases, resection of infarcted ovarian torsion (e.g. salpingo-oophorectomy) was performed and in four cases there was fenestration of large cysts. Using a LAMA approach in the case of symptomatic functional cysts, a transabdominal needle aspiration of the cyst was performed and the deflated cyst wall was exteriorized either through the umbilicus or through a $2 \mathrm{~cm}$ cosmetic incision in the hypogastric area (Figure 3). In 10 females with a cyst diameter $<5 \mathrm{~cm}$, a conservative approach was applied, and no further surgery was required.

Intestines: Enteric duplication cyst was diagnosed in six children (four males and two females). In all cases, surgery was applied: in four cases a LAMA approach was applied and classical open laparotomy was utilized in two cases. Although in the endoscopic view enteric duplication cysts do not clearly differ from the normal bowel wall, we found that proper bowel loop assessment starting from the level of the ileocecal valve in all cases revealed the location of the lesion using a LAMA approach. A mini-laparotomy allowed for excellent postoperative results (Figure 4). The anastomosis was performed extra-corporally through the umbilical wound or a small incision located directly over the lesion site. The treatment was based on enucleation of the enteric duplication with partial intestinal anastomosis $(n=4)$ or segmental resection of the intestine with primary anastomosis $(n=2)$.

Mesentery: Lymphatic mesenteric cysts were diagnosed in three male children. In each case, apart from ultrasound examination, computed tomographic scan was performed and laboratory markers were analyzed. Surgical treatment was delayed in each case, usually after the child finished 3 weeks of life. Laparotomy was followed by segmental intestinal resection in one localized case or subtotal resection of mesenteric pathological lesion. Histopathological examination confirmed lymphatic malformation or mesenteric cyst with solid and cystic component.

Other cysts: In the single case of splenic cyst, a conservative "watch and wait" approach was accepted, and the cyst underwent spontaneous regression. Pancreatic cysts located 

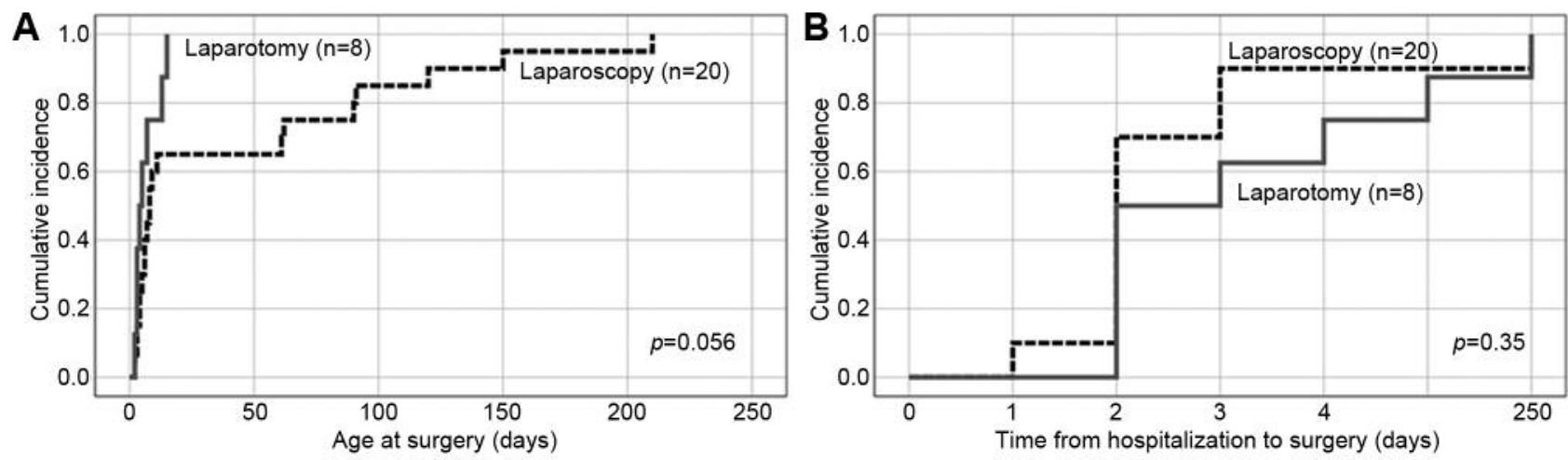

Figure 2. Surgical approach with respect to age at surgery (A) and time from hospitalization to surgery (B).

in the pancreatic head required diagnostic laparoscopy and after confirmation of true simple cystic character, the patients were followed-up and after the patients were 6 months old, definitive surgery was carried out because of dynamic growth of the lesion.

Complications. Postoperative bleeding requiring re-laparotomy occurred in two cases. In the first case, an enteric duplication was removed through laparotomy and because of cyst localization exactly at the level of the ileocecal valve, we attempted to preserve the valve, therefore the duplication cyst was enucleated. However, on postoperative day 2, the child presented with active bleeding from the gastrointestinal tract. During the reoperation, a temporary ileostomy was created, which was successfully closed after 3 months. The second patient underwent removal of the mesenteric cyst which was located in a technically demanding localization, immediately below the lower margin of the pancreas. The patient required re-laparotomy on postoperative day 1 because of bleeding from the small branch of the splenic vein. Both patients required transfusion of 1 unit of packed red blood cells, and no other complications were observed in either patient.

In one case of surgery for prenatal ovarian torsion, an accidental opening of the bladder occurred. It was primarily closed with a double layer of stitches without further consequences. There were no other complications related to the surgical procedure.

Postoperative course. Results of surgical treatment were very good. In each case, after surgical treatment, neonates were followed-up in an intensive care unit for at least 24 hours. No blood transfusion apart from two cases requiring re-laparotomy and no early complications were observed in any case; no death occurred.

A conservative approach in the case of small ovarian cysts $(<5 \mathrm{~cm}$ in diameter) and splenic cyst led to them resolving spontaneously. In all cases of surgical treatment, pathological examination was performed and no case of malignant transformation was found.

\section{Discussion}

A single-center experience of CACL management based on the application of abdominal ultrasound along with a laparoscopic approach is presented here. As no official standard management of neonates with congenital abdominal cystic lesions is available, we showed our therapeutic work-up, which has evolved during 8 years of clinical experience, in single-center analysis. This approach based on minimally invasive surgery proved to be safe and efficacious in preliminary diagnosis and differentiation of congenital abdominal cystic lesions.

In this analysis, we summarized and discussed characteristics and management of various types of CACL. The most often encountered abdominal cysts in our group of patients were ovarian cysts, intestinal duplications and mesenteric cysts.

The detection of CACL has risen dramatically with extended use of ultrasound examination (5). Furthermore, nowadays, the quality of available sonographic devices is much better; growing expertise allows for near-exact qualification of cystic lesions in terms of both origin and localization.

Typical distinctive characteristics of CACL on abdominal ultrasound examination for ovarian cysts include: location at one side of the bladder, round or oval-shaped and observed in female patients. We found useful a distinction of CACL of ovarian origin according to the criteria of Nussbaum et al. into uncomplicated cyst (anechogenic, unilocular, thin-walled) and complicated cysts (heterogenous with hyperechogenic components, thick-walled, containing free-floating material with intracystic septations) (6). We also found that the socalled 'daughter cysts sign' were also typical for functional ovarian cysts (7). In contrast to other authors, we found the absence of color Doppler flow not to be $100 \%$ specific for ovarian torsion (6). In our observation, a highly sensitive 
A

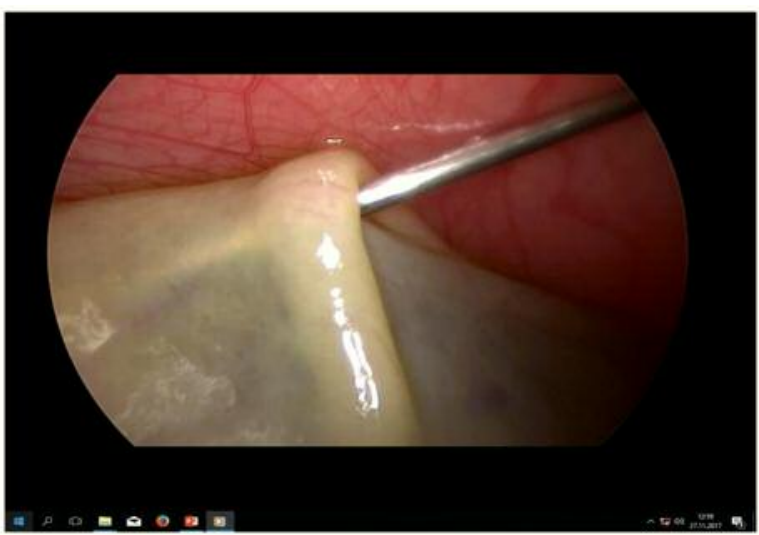

B

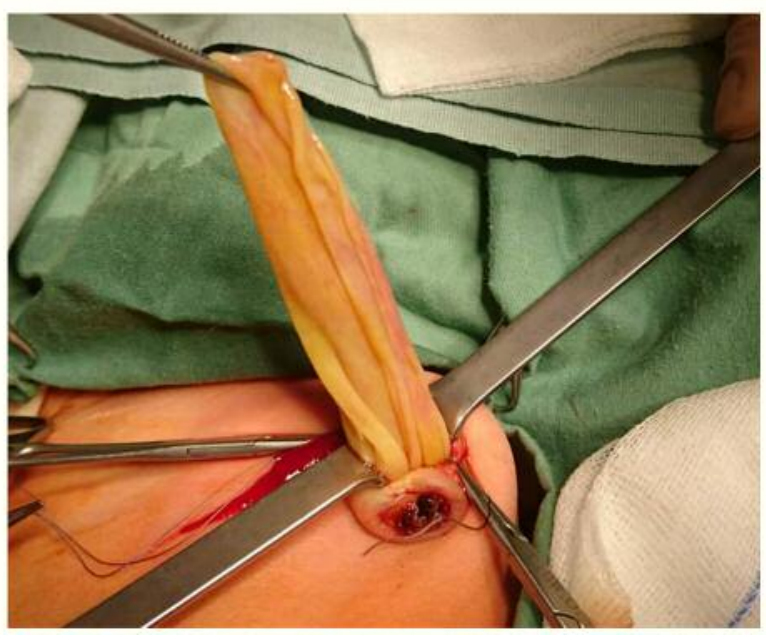

Figure 3. A: Intraoperative view of transabdominal ovarian cyst decompression under laparoscopic guidance. B: Exteriorization of deflated ovarian cyst through the umbilicus.

sonography is prone to display peripheral flow even in the case of ovarian torsion with accomplished infarction; most likely this phenomenon is due the 'foreign body reaction' with enhanced blood flow of the surrounding tissues in the course of infective complications. All patients with complicated ovarian cyst in our study were operated on and cyst diameter was more than $5 \mathrm{~cm}$; however, we observed that the most important for decision-making is not the diameter of the cyst (even compression causes change in maximum diameter) but the sonographic appearance of the lesion.

In the case of cystic intestinal duplications, they are located in the middle of abdomen, close to the intestine and have an intestinal wall-like structure. In our opinion, additional clinical suspicion important for sonographic diagnosis of enteric duplications is inconsistency in sequential examinations together with prenatal presence of the lesion. After finding a typical sonographic appearance of enteric cyst, we propose surgical removal in the planned setting irrespective of the diameter of the lesion. Possible

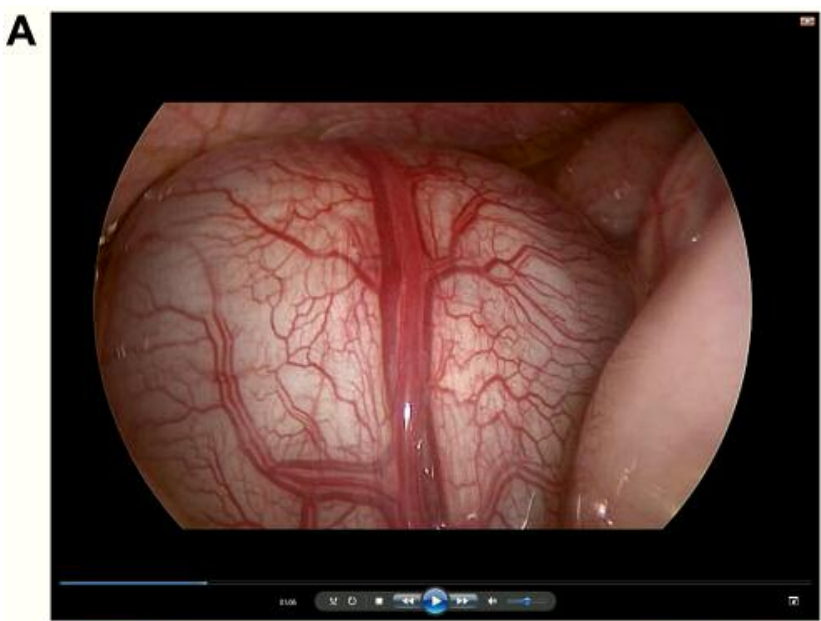

B

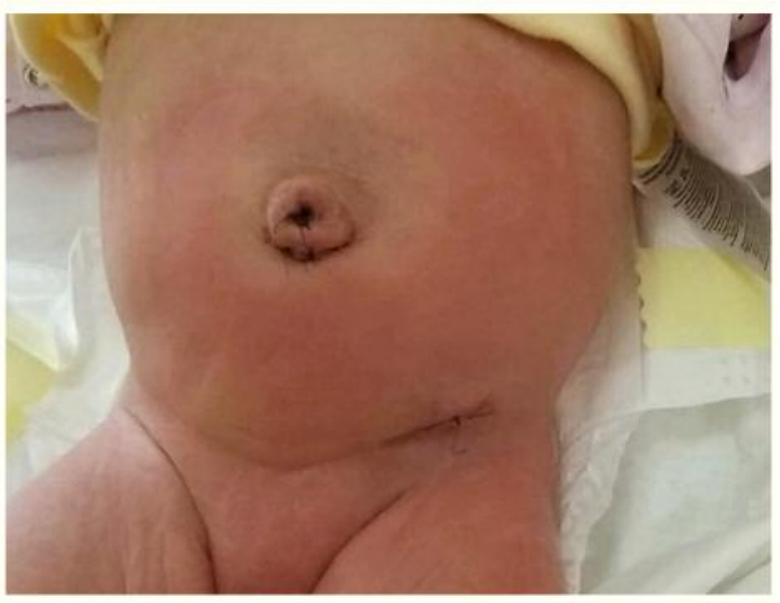

Figure 4. A: Endoscopic view of cystic enteric duplication. B: Postoperative day 1 , good cosmetic result after laparoscopically assisted minimal access approach, small scar in the hypogastric region is almost invisible.

complications include bowel obstruction or hemorrhage (8).

In our study, mesenteric cysts were round-shaped with a thin tensionless wall, or presenting multiple chambers, and were easily deformable on compression. The sonographic findings in our study demonstrated that a comprehensive analysis of mesenteric cysts via ultrasonography may provide an adequate differential diagnosis although due to the nature of the lesions, before surgical treatment, we advocate more precise radiological examinations. Computed tomography and magnetic resonance imaging can be helpful and essential in order to obtain better feature description of the mass (9). Additional information including the lesion origin, its relationships and its adhesion to visceral organs can be useful if laparoscopic surgery is being considered (10). Similarly to enteric duplication cysts, in the case of mesenteric cyst, we advocate surgical treatment. Abdominal pain is documented as the commonest symptom and an acute abdominal presentation revealing bowel obstruction is often reported in infants. Our 
experience also revealed that two patients with mesenteric cysts presented with an intestinal obstruction. Other reported complications include torsion, infarction, volvulus formation (11) or even perforation, infection, anemia from intracystic bleeding, and rarely rupture (12). Due to complex anatomic circumstances, we also preferred additional diagnostic modalities in the case of cystic pancreatic or duodenal lesions. With growing expertise in minimally invasive surgery, we do not advocate magnetic resonance examination in the case of CACL of suspected ovarian origin. The LAMA approach serves as good modality to perform diagnostic and therapeutic intervention. We agree that minimally invasive surgery with a laparoscopic approach should be the treatment of choice. In neonates, it requires an extremely high level of expertise for both the surgeon and anesthesiologists. With this approach, an improved outcome was shown in other diseases (13-15). Enhanced recovery after surgery (ERAS) or 'fast-track' methods enable standardization of postoperative medical care, improvement of patient outcomes, promotion of early recovery, and reduction of healthcare expenditure. Fast-track surgery is a multifunctional concept involving pre-, peri- and postoperative measures aiming to reduce the length of hospital stay and morbidity and complication rates, following elective abdominal surgery (15).

In conclusion, our strategy of management in the analyzed group of newborns and young infants with CACL based on a LAMA approach allowed for the safe treatment of the vast majority of cases, with an acceptable complication rate and effective surgical treatment with good cosmetic results.

\section{Conflicts of Interest}

The Authors have no conflicts of interest to disclose in regard to this study.

\section{Authors' Contributions}

PG: Concept/design, data analysis/interpretation, writing article; PG, $\mathrm{KR}, \mathrm{KK}$, and JS: data collection, data analysis/interpretation; PG, JS: critical revision of article, approval of article.

\section{References}

1 Silva CT, Engel C, Cross SN, Copel JE, Morotti RA, Baker KE and Goodman TR: Postnatal sonographic spectrum of prenatally detected abdominal and pelvic cysts. Am J Roentgenol 203(6): W684-696, 2014. PMID: 25415735. DOI: 10.2214/AJR. 13.12371

2 Ozcan HN, Balci S, Ekinci S, Gunes A, Oguz B, Ciftci AO and Haliloglu M: Imaging findings of fetal-neonatal ovarian cysts complicated with ovarian torsion and autoamputation. Am J Roentgenol 205(1): 185-189, 2015. PMID: 26102397. DOI: 10.2214/AJR.14.13426

3 Aydin BK, Saka N, Bas F, Yilmaz Y, Haliloglu B, Guran T, Turan S, Bereket A, Yesiltepe Mutlu G, Cizmecioglu F, Hatun S, Bezen D, Tutunculer F, Cebeci N, Isguven P, Memioglu N,
Ercan O, Poyrazoglu S, Bundak R and Darendeliler F: Evaluation and treatment results of ovarian cysts in childhood and adolescence: A multicenter, retrospective study of 100 patients. J Pediatr Adolesc Gynecol 30(4): 449-455, 2017. PMID: 28167139. DOI: 10.1016/j.jpag.2017.01.011

4 Galazka P, Czyzewski K, Marjanska A, Daniluk-Matras I and Styczynski J: Minimally invasive surgery in pediatric oncology: Proposal of guidelines. Anticancer Res 39(11): 5853-5859, 2019. PMID: 31704809. DOI: 10.21873/anticanres.13789

5 Ibrahim H, Lewis D, Harrison GK, Tice H and Sangster G: Congenital ovarian cyst. J Perinatol 27(8): 523-526, 2007. PMID: 17653221. DOI: 10.1038/sj.jp.7211760

6 Nussbaum AR, Sanders RC, Hartman DS, Dudgeon DL and Parmley TH: Neonatal ovarian cysts: Sonographic-pathologic correlation. Radiology 168(3): 817-821, 1988. PMID: 3043551. DOI: $10.1148 /$ radiology.168.3.3043551

7 Lee HJ, Woo SK, Kim JS and Suh SJ: "Daughter cyst" sign: A sonographic finding of ovarian cyst in neonates, infants, and young children. Am J Roentgenol 174(4): 1013-1015, 2000. PMID: 10749241. DOI: 10.2214/ajr.174.4.1741013

8 Ranganath SH, Lee EY and Eisenberg RL: Focal cystic abdominal masses in pediatric patients. Am J Roentgenol 199(1): W1-16, 2012. PMID: 22733917. DOI: 10.2214/AJR.11.6642

9 Belhassen S, Meriem B, Rachida L, Nahla K, Saida H, Imed K, Sana M, Amine K, Lassad S, Mongi M, Mohsen B and Abdellatif N: Mesenteric cyst in infancy: Presentation and management. Pan Afr Med J 26: 191, 2017. PMID: 28674584. DOI: $10.11604 /$ pamj.2017.26.191.11476

10 Lee DL, Madhuvrata P, Reed MW and Balasubramanian SP: Chylous mesenteric cyst: A diagnostic dilemma. Asian J Surg 39(3): 182-186, 2016. PMID: 23769746. DOI: 10.1016/ j.asjsur.2013.04.009

11 Karim T, Topno M and Kate M: Simple mesenteric cyst in a child: Presentation and management. Arab J Gastroenterol 12(2): 90-91, 2011. PMID: 21684480. DOI: 10.1016/j.ajg.2010.10.007

12 Takeuchi K, Takaya Y, Maeda K and Maruo T: Peritonitis caused by a ruptured, infected mesenteric cyst initially interpreted as an ovarian cyst. A case report. J Reprod Med 49(1): 65-67, 2004. PMID 14976800

13 Ratiu D, Luncescu C, Morgenstern B, Eichler C, Gruttner B, Ludwig S, Mallmann $\mathrm{P}$ and Thangarajah F: Comparison of minimally invasive surgery and abdominal surgery among patients with cervical cancer. Anticancer Res 39(5): 2661-2664, 2019. PMID. 31092465. DOI: 10.21873/anticanres. 13390

14 Papazarkadas X, Spartalis E, Patsouras D, Ioannidis A, Schizas D, Georgiou K, Dimitroulis D and Nikiteas N: The role of 3D printing in colorectal surgery: Current evidence and future perspectives. In Vivo 33(2): 297-302, 2019. PMID: 30804106. DOI: 10.21873 /invivo.11475

15 Garmpis N, Dimitroulis D, Garmpi A, Diamantis E, Spartalis E, Schizas D, Angelou A, Margonis GA, Farmaki P, Antoniou EA, Mantas D, Markatos K, Kontzoglou K and Damaskos C: Enhanced recovery after surgery: Is it time to change our strategy regarding laparoscopic colectomy? In Vivo 33(3): 669674, 2019. PMID: 31028183. DOI: 10.21873/invivo.11525

Received February 24, 2020 Revised March 10, 2020 Accepted March 12, 2020 